\title{
Amphotericin B for cryptococcal meningitis in HIV positive patients: Low dose versus high dose
}

\author{
S. Rajeshwari, Prabha M. R. Adhikari*, John T. Ramapuram*, Satish Rao*, M. R. S. M. Pai, Kiran*
}

Aim: To compare the safety and efficacy of low dose vs high dose of amphotericin B in cryptococcal meningitis associated with HIV infection. Materials and Methods: Retrospective data of patients admitted with clinical diagnosis with or without microbiological evidence of cryptococcal meningitis was collected from Jan 2000Mar 2006. Patients' details were collected in a proforma which included patient's age, weight, signs and symptoms of disease and microbiological report (blood and CSF analysis). Data also included coexisting disease; concomitant medications taken along with amphotericin B. Adverse drug reactions which occurred during the period of treatment were recorded. Patients were grouped as low dose group and high dose group depending on the dose of amphotericin B given for the treatment of cryptococcal meningitis. Patients who received amphotericin $B$ at doses of 0.33 to $0.64 \mathrm{mg} / \mathrm{kg}$ body weight per day were categorized under low dose group and patients who received amphotericin $B$ at doses of 0.7 to $1.1 \mathrm{mg} / \mathrm{kg} /$ day were categorized under high dose group. All data were pooled and analyzed between the groups using chi square test. Result: Total number of patients included in the study were 38, 26 in the low dose group and 12 in the high dose group. In the low dose group, 20 were males and six were females, in the high dose group eight were males and four were females. The commonest underlying diseases were tuberculosis (17 in low dose group, nine in high dose group), Pneumocystis carinii (jeroveci) pneumonia (16 in low dose group, seven in high dose group) and oral candidiasis (eight in low dose group, seven in high dose group), Toxoplasmosis (three in low dose group, one in high dose group), hypertension (1 in group A) and diabetes mellitus (1 in group B). Concomitant medication received along with amphotericin B for coexisting diseases in both the groups were antitubercular therapy, cotrimoxazole, antiviral therapy and premedications such as Ondansetran, Domperidone, Diclofenac, Mannitol, Dexamethazone and Pheniramine. Comparison between the groups showed that the cure rate is similar in both the groups $(P=0.440$, where as over all mortality was higher in low dose group than in high dose group which was statistically significant $(P=0.03)$. Adverse effects were higher in high dose group than in low dose group such as hypokalemia $(P=0.04)$, facial puffiness $(P=0.01)$. Other adverse effects were comparable in both the groups. Conclusion: High dose of amphotericin $B$ therapy is more efficacious. However hypokalemia and clinical features of nephrotoxicity was higher with patients on high dose therapy, which can be managed by proper monitoring.

Key words: Amphotericin B, cryptococal meningitis, human immunodeficiency virus, hypokalemia

From:

Departments of Pharmacology and *Medicine, Kasturba Medical College,

Mangalore, India

Correspondence:

Dr. S. Rajeshwari, Department of Pharmacology, Kasturba Medical College, Light House Hill Road, Managalor - 575 001, Karnataka, India.

E-mail: rajeashwarishasrty@yahoo.co.in

\section{Introduction}

Cryptococcosis is an infectious disease caused by Cryptococcus neoformans, an encapsulated yeast. Most patients with acquired immune deficiency syndrome (AIDS) who are infected with C.neoformans develop 
meningitis. ${ }^{[1]}$ Cryptococcal meningitis $(\mathrm{CM})$ is still the most common manifestation of systemic fungal infection in human immunodeficiency virus (HIV) infected patients and remains associated with significant morbidity and mortality. ${ }^{[2]}$ Common presenting symptoms are severe headache; fever, progressive malaise, nausea with vomiting, fatigue, blurred vision and / or sensitivity to bright light, stiff neck, altered behavior changes, coma. ${ }^{[3]}$ The overall incidence of cryptococcal meningitis in patients with AIDS appears to be $5-10 \% .{ }^{[4]}$ Recent data indicates that incidence of C.neofomans is high in developing countries. ${ }^{[5]}$ Cryptococcal meningitis has been reported as the most common opportunistic infection of the CNS of Indian patients with HIV. ${ }^{[6]}$

Untreated $\mathrm{CM}$ is fatal with very rare exception. ${ }^{[7]}$ For more than 40 years, conventional amphotericin $B$ deoxycholate $(\mathrm{AMB})$ has been considered to be the gold standard for initial treatment of CM in patients with HIV infection, ${ }^{[2]}$ because of its broad fungicidal activity and cheapness. Treatment with AMB however is associated with acute reactions related to infusion and dose dependent nephrotoxicity. ${ }^{[8]}$

\section{Objective}

To compare the safety and efficacy of low dose versus high dose of amphotericin B in cryptococcal meningitis associated with HIV infection.

\section{Materials and Methods}

A retrospective chart review of patients hospitalized with clinical diagnosis, with or without microbiological evidence of cryptococcal meningitis was collected from Jan 2000- March 2006. Cerebrospinal fluid (CSF) was studied in these patients. Laboratory investigation of CSF was traditionally based on microscopic examination of Gram's staining, culture and India ink preparations. Patients' details were collected in a patient proforma including patient's age, weight, signs and symptoms of disease and microbiological report (Blood and CSF). Data also included other comorbidities and concomitant medications taken along with AMB. Adverse drug reactions which occurred during the period of $A M B$ treatment were recorded.

Patients were grouped into two groups: low dose group and high dose group, depending on the dose of AMB given for the treatment of $\mathrm{CM}$. Patients who received
$\mathrm{AMB}$ at doses of 0.33 to $0.64 \mathrm{mg} / \mathrm{kg} /$ day were categorized under low dose group and patients who received AMB 0.7 to $1.1 \mathrm{mg} / \mathrm{kg} / \mathrm{day}^{\left[{ }^{9-11]}\right.}$ were categorized under high dose group. All the data were pooled and analyzed between the groups using Chi square test.

\section{Result}

Total numbers of subjects included in the study were 38 patients. Among these 26 were in low dose group and 12 were in high dose group. There was a male preponderance in this study.

The demographic data of patients are as shown in the Table 1.

Typical presentations of $\mathrm{CM}$ in these patients were as shown in the Table 2. The most common signs and symptoms were headache, fever, nausea/vomiting, neck stiffness, cough and behavioral changes etc.

CM was associated with tuberculosis (TB) (26/38 cases), Pneumocystis carinii pneumonia (jeroveci) (23/38 cases) oral candidiasis (OC) (15/38 cases), toxoplasmosis (TXG) (4/38 case), hypertension (HTN) (1/36 case) and diabetes mellitus (DM) (1/38 case). There were no statistical difference in coexisting disease between the two groups [Figure 1].

The concomitant medication received along with AMB for comorbid conditions were anti-tubercular therapy (ATT), pyridoxine, cotrimoxazole, clotrimazole, sulphadoxine+ pyrimethamine, enalapril and insulin. Antiretroviral therapy was noted in 24 patients, 14 in the low dose group and 10 in the high dose group.

Among the 26 patients from low dose group, 3three patients were empirically treated $\mathrm{CM}$ for 14 days. Two

\begin{tabular}{lc}
\hline Table 1: Demographic data & \\
\hline Characteristic & Value $\mathbf{n}=\mathbf{3 8}$ \\
Male & 28 \\
Female & 10 \\
Age $(Y r)$ & \\
$\quad$ Median & 33.5 \\
Range & $20-50$ \\
Weight (Kg) & \\
$\quad$ Median & 48.50 \\
$\quad$ Range & $30-70$ \\
CD4 count (cells/micro L) & \\
$\quad$ Median & 104 \\
Range & $15-323$ \\
\hline
\end{tabular}


Table 2: Signs and symptoms of cryptococcal meningitis

\begin{tabular}{lccc}
\hline Signs and symptoms & Low dose $(<\mathbf{0 . 7} \mathbf{~ m g / k g / d a y )}(\mathbf{n}=\mathbf{2 6})$ & High dose $(\geq \mathbf{0 . 7} \mathbf{~ m g / k g / d a y})(\mathbf{n}=\mathbf{1 2})$ & $P$ value \\
Headache & 23 & 11 & 0.76 \\
Fever & 19 & 8 & 0.69 \\
Nausea/vomiting & 17 & 8 & 0.93 \\
Neck stiffness & 12 & 10 & $0.03^{*}$ \\
Cough & 5 & 2 & 0.85 \\
Behavioral changes & 3 & 1 & 0.76 \\
Papilledema & 3 & 1 & 0.76 \\
Unconsciousness & 3 & 0 & 0.22 \\
Convulsions & 2 & 0 & 0.33 \\
Hepatomegaly & 1 & 1 & 0.56 \\
Visual changes & 4 & 0 & 0.15 \\
Giddiness & 2 & 0 & 0.32 \\
\hline
\end{tabular}

( ${ }^{\star} P$ value is calculated using Chi square test)

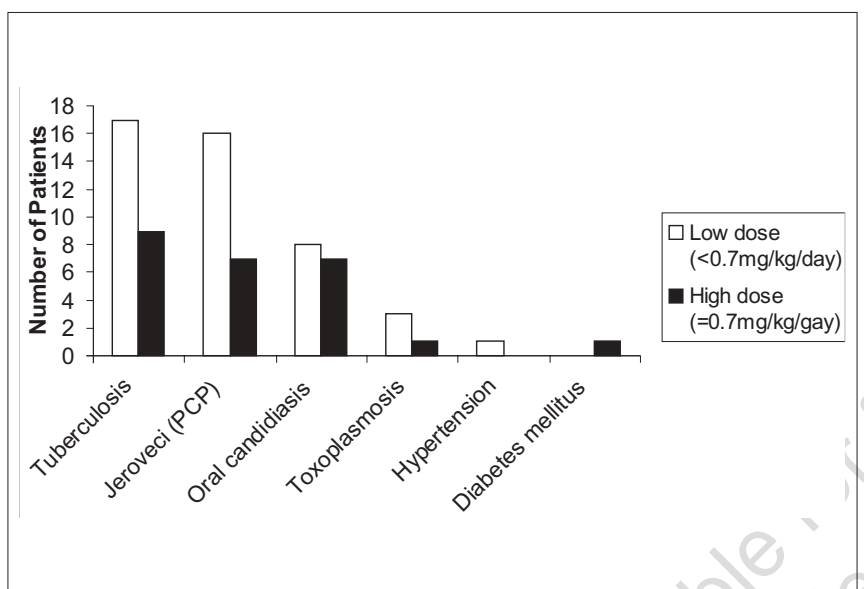

Figure 1: Coexisting disease in AIDS associated with CM

patients were discharged against medical advice on the fourth and ninth day of AMB treatment. Among the 21 patients, 15 patients completed treatment. Of these 15 patients, 14 patients were cured completely but one patient remained microbiologically positive for $\mathrm{C}$.neoforman after 14 days of therapy. Six patients expired between the first and the ninth day, after initiation of amphotericin B treatment (23.07\% patients). Among the 15 patients from low dose group, relapse was seen in four patients, of these two patients expired. The overall mortality of $30.76 \%$ (8/26 cases) was seen in the low dose group.

Twelve patients were categorized under high dose group. Of these, three were discharged against medical advice. Among the nine patients who completed treatment, two who showed clinical improvement remained microbiologically positive by India ink preparation for C.neoforman after 14 days of therapy. Seven patients showed complete cure. No patients were treated empirically. Neither relapse or nor mortality was reported in group B. Table 3 shows comparison of efficacy between the two groups.
Table 3: Comparison of efficacy between the two groups

Parameters
Low dose High dose ( $<0.7 \mathrm{mg} / \mathrm{kg} / \mathrm{day}) \quad(\geq 0.7 \mathrm{mg} / \mathrm{kg} / \mathrm{day})$

Empirical treatment ( $\mathrm{n}$ ) 3 0 Discharge against medical advice (n)

Complete cure rate $(n)$ India ink positive (n)

Expired (n)

Relapse ( $\mathrm{n}$ )

Overall mortality $(\mathrm{n})$

( $\left.{ }^{\star} P=0.02\right)$

Table 4: Comparison of adverse event

\begin{tabular}{lccc}
\hline Adverse event & $\begin{array}{c}\text { Group A } \\
(\mathbf{n}=\mathbf{2 6})\end{array}$ & $\begin{array}{c}\text { Group B } \\
(\mathbf{n}=\mathbf{1 2})\end{array}$ & $\boldsymbol{P}$ value \\
Fever, chills, rigor & 11 & 6 & 0.66 \\
Hypokalemia & 10 & 9 & $0.04^{*}$ \\
Renal dysfunction & 6 & 2 & 0.65 \\
Thrombophlebitis & 6 & 4 & 0.05 \\
Vomiting & 9 & 5 & 0.67 \\
Seizure & 5 & 1 & 0.39 \\
Hyponatremia & 3 & 0 & 0.22 \\
Facial puffiness & 0 & 4 & $0.01^{*}$ \\
\hline
\end{tabular}

$P$ values were determined by the chi-square test, ${ }^{*} P<0.05$ statistically significant

The commonest adverse effects were fever, chills with rigor (17/38 cases), vomiting (14/38 cases), hypokalemia (19/38 cases), thrombophlebitis ( $10 / 38$ cases), increased serum creatinine ( $8 / 38$ cases), seizures ( $6 / 38$ cases), hyponatremia ( $3 / 38$ cases), facial puffiness ( $4 / 38$ cases). Comparisons between the two groups [Table 4] showed that hypokalemia and facial puffiness were more in high dose group which was statistically significant $(P=0.04$ and $P=0.01$ respectively). All other adverse effects were comparable between the two groups.

\section{Discussion}

Cryptococcal meningitis is increasing because of rising population of immunocompromized individuals particularly those with AIDS. ${ }^{[5]}$ Fungal infections are a 
major cause of morbidity and mortality in HIV. Untreated cryptococcal meningitis is fatal.

The recommended initial treatment for acute disease is amphotericin B for two weeks duration. Main limitation in the use of $A M B$ is its toxicity. Acute infusion-related reactions fever, chills with rigors and hypokalemia. In our study also it has been seen that infusion related side effects like fever, chills with rigor, vomiting are common. Pre-medication before receiving AMB can help to prevent/ reduce some side effects during infusion. ${ }^{[12]}$ In our study, pretreatment with antipyretics, antihistaminics and corticosteroids has helped to lessen these effects.

AMB can also cause significant electrolyte abnormalities (hypokalemia and hypomagnesaemia). These effects occur in the majority of patients within the first week of therapy. Electrolytes should be monitored daily and replaced as needed. ${ }^{[13]}$ Hypokalemia and hyponatremia are seen in the present study. This electrolyte imbalance is corrected by giving appropriate electrolytes and fluids. Patients treated with AMB should be monitored for dose dependent nephrotoxicity. In our study there was a increased serum creatinine observed in both the groups. But facial puffiness was predominantly observed in high dose therapy group $(P=0.01)$, which may be indicative of a dose-dependent nephrotoxicity.

Diagnosis of invasive fungal infections early, reliably and definitively continues to be a major challenge to practitioners. As a result, empiric therapy is generally initiated in response to a persistent fever in a vulnerable patient. ${ }^{[14]}$ The same trend was seen in the present study also. Although CSF did not shown positive results for cryptococcal meningitis, three out of 38 patients $(7.89 \%$ cases) who presented with intense headache, signs of meningitis with low $\mathrm{CD}_{4}$ cell count and marginally elevated protein where treated empirically.

A potential strategy for reducing AMB associated toxicity is the use of liposomal and other lipid- based formulations containing AMB. ${ }^{[15,16]}$ These formulations have shown a reduced toxicity and have permitted the use of higher doses of AMB, which potentially could lead to an increased therapeutic index. ${ }^{[17]}$ These so called liposomal formulations of $A M B$ are very expensive, thus limiting their use. Drug acquisition costs for all these products are much higher than for conventional AMB.
Treatment with a low daily dose of AMB in AIDS patients with cryptococcal meningitis has been associated with low efficacy and high mortality. ${ }^{[18]}$ In the present study, high mortality was seen in the low dose therapy group $(30.76 \%)$ when compared to the high dose therapy group.

The standard recommended dose for $\mathrm{AMB}$ for the treatment of cryptococcal meningitis is 0.7 to $1.0 \mathrm{mg} / \mathrm{kg} /$ day. ${ }^{[19]}$ In view of the cost of therapy and fear of adverse events clinicians were using low dose for patients who could not afford high dose and who had poor nutritional status.

Results of our study clearly showed that low dose therapy was associated with a significant high mortality (8 versus 0 with a $P$ value $<0.02$ ) and higher relapse rate although this was not statistically significant because of the small number in the high dose group. Occurrence of hypokalemia was significantly high in high dose group however this was easily correctable. Puffiness of face is significantly high in patients on high dose as compared to low dose indicating sub-clinical renal dysfunction.

All the side effects were easily treated and none of them life-threatening. Hence fear of side effect should not be a cause of concern for the physicians to prescribe high dose of $\mathrm{AMB}$ for $\mathrm{CM}$.

Use of potent antiretroviral therapies results in a decrease in the incidence of opportunistic infections associated with AIDS. ${ }^{[20]}$ Highly active anti-retroviral therapy has changed the face of HIV/AIDS by leading to a dramatic decrease in HIV related morbidity and mortality among those with accesses to therapy. ${ }^{[21]}$ In this study, more number of patients in the high dose group received anti-retroviral therapy when compared to low dose group $(P=0.08)$. Obviously the outcome of therapy resulting in higher efficacy for the high dose group.

\section{Conclusion}

High dose of amphotericin B therapy is more efficacious. However hypokalemia and clinical features of nephrotoxicity was higher with patients on high dose therapy, which can be managed by proper monitoring.

\section{References}

1. Sar B, Monely D, Vann M, Keo C, Sarthou JL, Buisson Y. 
Increasing invitro resistance in Cryptococcus neoformans Cambodian isolates: April 2000-to 2002. J Antimicrob Chemother 2004;54:563-5.

2. Leenders AC, Reiss P, Portegies P, Clezy K, Hop WC, Hoy J, et al. Liposomal amphotericin B compared with amphotereicin B both followed by oral fluconazole in the treatment of AIDS-associated cryptococcal meningitis. AIDS 1997;11:1463-71.

3. Philips, Peter. "Fungal infections in AIDS patients". Grand Rounds Infect Dis 1994;4:5-11.

4. Hajjeh RA, Conn LA, Stephens DS, Baughman W, Hamill R, Graviss E, et al. Cryptococcosis: Population-based multi-state active surveillance and risk factors in human immunodeficiency virus infected persons. Cryptococcal Active Surveillance Group. J Infect Dis 1999;179:449-54.

5. Khanna N, Chandramuki A, Desai A, Ravi V, Santosh V, Shankar $\mathrm{SK}$, et al. Cryptococcosis in the immunocompromized host with special reference to AIDS. Indian J Chest Dis Allied Sci 2000;42:311-5.

6. Kumarasamy N, Vallabhaneni S, Flanigan TP, Mayer KH, Solomon S. Clinical profile of HIV in India. Indian J Med Res 2005;121:37794.

7. Rippon JW. Cryptococcosis. In. Medical mycology. The pathogenic Fungi and the Pathogenic Actinomycetes. $3^{\text {rd }}$ ed. Saunders: Philadelphia; 1998. p. 582-609.

8. Eriksson U, Seifert B, Schaffner A. Comparison of effects of amphotericin B deoxycholate infused over 4 to $24 \mathrm{hrs}$ : Randomized controlled trial. BMJ 2001;322:579-82.

9. Pitisuttithum P, Tansuphasawadikal S, Simpson AJ, Howe PA White NJ. A prospective study of AIDS- associated cryptococcal meningitis in Thailand treatment with high dose amphotericin $B$. J Infect 2001;43:226-33.

10. van.der Horst CM, Saag MS, Cloud GA, Hamill RJ, Graybill JR, Sobel JD, et al. Treatment of cryptococcal meningitis associated with the acquired immunodeficiency syndrome. National Institute of Allergy and Infectious Diseases Mycoses Study Group and AIDS Clinical Trials Group. N Engl J Med 1997;337:15-21.

11. Saag MS, Powderly WG, Cloud GA, Robinson P, Grieco MH,
Sharkey PK, et al. Comparision of amphotericin B with fluconazole in the treatment of acute ADIS-associated cryptococcal meningitis. N Engl J Med 1992;326:83-9.

12. Walsh TJ, Gonzalez C, Lyman CA, Chanock SJ, Pizzo PA. Invasive fungal infections in children: Recent advances in diagnosis and treatment. Adv Pediatr Infect Dis 1996;11:187-290.

13. Peacock JE, Herrington DA, Cruz JM. Amphotericin B therapy: Past, present and future. Infect Dis Clin Pract 1993;2:81-93.

14. Hughes WT, Armstrong D, Bodey GP, Bow EJ, Brown AE, Calandra T, et al. 2002 guidelines for the use of antimicrobial agents in neutropenic patients with cancer. Clin Infect Dis 2002;34:730-51.

15. Janknegt R, Marie de S, Bakker Woundenberg IA, Crommelin JA. Liposomal and lipid formulations of amphotericin B: Clinical pharmacokinetics. Clin Pharmacokinet 1992;23:279-91.

16. Graybill JR. Lipid formulations of amphotericin B: Does the emperor need new cloths? Ann Intern Med 1996;124:921-3.

17. Leenders AC, de Marie $S$. The use of lipid formulations of amphotericin B for systemic fungal infections. Leukemia 1996;10:1570-5.

18. Hsieh SM, Hung CC, Chen MY, Hsueh PR, Chang SC. Efficacy and adverse effects of higher dose amphotericin B monotherapy for cryptococcal meningitis in patients with advanced HIV infection. J Microbiol Immunol Infect 1998;31:233-9.

19. Benett JE. Cryptococosis. In: Kasper DL, Braunwald E, Fauci AS, Hauser SL, Longo DL, Jameson JL, editors. Principles of internal medicine. $16^{\text {th }}$ ed. McGraw Hill: 2005. p. 1183-5.

20. Currie BP, Casadevall A. Estimation of the prevalence of cryptococcal infection among patients infected with the human immunodeficiency virus in New York city. Clin Infect Dis 1994;19:1029-33.

21. Palella FJ Jr, Delaney KM, Moorman AC, Loveless MO, Fuhrer J, Satten GA, et al. Declining morbidity and mortality among patients with advanced HIV infection. N Engl J Med 1998;338:853-60.

Source of Support: Nil, Conflict of Interest: None declared. 